\title{
Inhibition of Hemangiogenesis and LymphangiogenesisafterNormal-Risk Corneal Transplantation by Neutralizing VEGF Promotes Graft Survival
}

\section{Citation}

Cursiefen,Claus, Jingtai Cao, Lu Chen, Ying Liu, Kazuichi Maruyama, David Jackson, Friedrich

E. Kruse, Stanley J. Wiegand, M. Reza Dana, and J. Wayne Streilein. 2004. "Inhibition of Hemangiogenesis and LymphangiogenesisafterNormal-Risk Corneal Transplantation by Neutralizing VEGF Promotes Graft Survival." Investigative Opthalmology \& Visual Science 45 (8) (August 1): 2666. doi:10.1167/iovs.03-1380.

\section{Published Version}

doi:10.1167/iovs.03-1380

\section{Permanent link}

http://nrs.harvard.edu/urn-3:HUL.InstRepos:34388126

\section{Terms of Use}

This article was downloaded from Harvard University's DASH repository, and is made available under the terms and conditions applicable to Other Posted Material, as set forth at http:// nrs.harvard.edu/urn-3:HUL.InstRepos:dash.current.terms-of-use\#LAA

\section{Share Your Story}

The Harvard community has made this article openly available.

Please share how this access benefits you. Submit a story.

\section{Accessibility}




\title{
Inhibition of Hemangiogenesis and Lymphangiogenesis after Normal-Risk Corneal Transplantation by Neutralizing VEGF Promotes Graft Survival
}

\author{
Claus Cursiefen, ${ }^{1,2,3}$ Jingtai Cao, ${ }^{4}$ Lu Chen, ${ }^{1}$ Ying Liu, ${ }^{1}$ Kazuichi Maruyama, ${ }^{1}$ \\ David Jackson, ${ }^{5}$ Friedrich E. Kruse, ${ }^{3}$ Stanley J. Wiegand, ${ }^{4}$ M. Reza Dana, ${ }^{1}$ and \\ J. Wayne Streilein ${ }^{1,6}$
}

Purpose. To evaluate the occurrence and time course of hemand lymphangiogenesis after normal-risk corneal transplantation in the mouse model and to test whether pharmacologic strategies inhibiting both processes improve long-term graft survival.

Methods. Normal-risk allogeneic (C57BL/6 to BALB/c) and syngeneic (BALB/c to BALB/c) corneal transplantations were performed and occurrence and time course of hem- and lymphangiogenesis after keratoplasty was observed, by using double immunofluorescence of corneal flatmounts (with CD31 as a panendothelial and LYVE-1 as a lymphatic vascular endothelium-specific marker). A molecular trap designed to eliminate VEGF-A (VEGF Trap R1R2 $_{1} ; 12.5 \mathrm{mg} / \mathrm{kg}$ ) was tested for its ability to inhibit both processes after keratoplasty and to promote long-term graft survival (intraperitoneal injections on the day of surgery and 3, 7, and 14 days later).

RESults. No blood or lymph vessels were detectable immediately after normal-risk transplantation in either donor or host cornea, but hem- and lymphangiogenesis were clearly visible at day 3 after transplantation. Both vessel types reached donor tissue at 1 week after allografting and similarly after syngeneic grafting. Early postoperative trapping of VEGF-A significantly reduced both hem- and lymphangiogenesis and significantly improved long-term graft survival (78\% vs. $40 \% ; P<0.05)$.

Conclusions. There is concurrent, VEGF-A-dependent hem- and lymphangiogenesis after normal-risk keratoplasty within the preoperatively avascular recipient bed. Inhibition of hem- and lymphangiogenesis (afferent and efferent arm of an immune

From ${ }^{1}$ The Schepens Eye Research Institute, Department of Ophthalmology, Harvard Medical School, Boston, Massachusetts; ${ }^{4}$ Regeneron Pharmaceuticals Inc., Tarrytown, New York; the ${ }^{5}$ Medical Research Council Human Immunology Unit, Institute of Molecular Medicine, Oxford, United Kingdom; and ${ }^{3}$ the Department of Ophthalmology, Friedrich-Alexander-University Erlangen-Nürnberg, Erlangen, Germany.

${ }^{2}$ Present affiliation: Department of Ophthalmology, Friedrich-Alexander-University Erlangen-Nürnberg, Erlangen, Germany.

${ }^{6}$ Deceased March 15, 2004

Supported by Deutsche Forschungsgemeinschaft (German Research Foundation) Grants $\mathrm{Cu} 47 / 1-1$ and $\mathrm{Cu} 47 / 1-2$, and National Eye Institute Grant EY10765.

Submitted for publication December 19, 2003; revised April 5, 2004; accepted April 13, 2004.

Disclosure: C. Cursiefen, None; J. Cao, Regeneron Pharmaceuticals Inc. (F); L. Chen, None; Y. Liu, None; K. Maruyama, None; D. Jackson, None; F.E. Kruse, None; S.J. Wiegand, Regeneron Pharmaceuticals Inc. (E, F); M.R. Dana, None; J.W. Streilein, None

The publication costs of this article were defrayed in part by page charge payment. This article must therefore be marked "advertisement" in accordance with 18 U.S.C. $\$ 1734$ solely to indicate this fact.

Corresponding author: Joan Stein-Streilein, Schepens Eye Research Institute, Harvard Medical School, 20 Staniford Street, Boston, MA 02114; jstein@vision.eri.harvard.edu. response) after normal-risk corneal transplantation improves long-term graft survival, establishing early postoperative hemand lymphangiogenesis as novel risk factors for graft rejection even in low-risk eyes. (Invest Ophthalmol Vis Sci. 2004;45: 2666-2673) DOI:10.1167/iovs.03-1380

Corneal transplantation is the oldest, most successful, and most commonly performed tissue transplantation, with nearly 40,000 transplantations a year alone in the United States. ${ }^{1}$ When corneal grafts are placed into an avascular recipient bed (so-called normal-risk keratoplasty), 2-year graft survival rates approach $90 \%$ under cover of topical steroids, even without HLA-matching. ${ }^{2}$ This very successful outcome is attributable to corneal immune privilege (i.e., the phenomenon of suppressed corneal inflammation induced by an array of endogenous mechanisms that downregulate alloimmune and inflammatory responses in the cornea and its bed). These mechanisms include the lack of both afferent lymphatic and efferent blood vessels in the normal-risk recipient cornea, lack of MHC $\mathrm{II}^{+}$antigen-presenting cells (APCs), FASL-expression on corneal epithelium and endothelium, and the anterior chamber associated immune privilege (ACAID) directed at graft antigens, for example (for review see Ref. 1). In contrast, survival rates of corneal grafts placed into vascularized, not immuneprivileged recipient beds (so called high-risk keratoplasty) decrease significantly to below 50\% (even with local and systemic immune suppression). ${ }^{3,4}$ Preexisting corneal stromal blood vessels have been identified as strong risk factors for immune rejection after corneal transplantation, both in the clinical setting ${ }^{4}$ and in the well-defined mouse model of corneal transplantation. ${ }^{5}$ Recently, in addition to blood vessels, biomicroscopically undetectable lymphatic vessels have been found in association with blood vessels in vascularized high-risk human corneas, ${ }^{6,7}$ and it is likely that corneal lymphatic vessels enable effective access of donor and host APCs and antigenic material to regional lymph nodes where accelerated sensitization to graft antigens occurs. ${ }^{8}$

But even in the normal-risk setting (with a preoperatively avascular recipient bed), mild corneal hemangiogenesis develops after keratoplasty ${ }^{9-11}$ : Outgrowth of new blood vessels from the limbal arcade toward the graft can be observed within the first postoperative year in approximately $50 \%$ of patients undergoing normal-risk keratoplasty, and in $10 \%$ of patients these new blood vessels even reach the interface or invade donor tissue ${ }^{11}$ at corneal suture sites and then proceed centrally. ${ }^{9-11}$

Both hem- and lymphangiogenesis (i.e., the outgrowth of new blood vessels versus lymphatic vessels from preexisting vessels) are mediated by members of the VEGF growth factor family: VEGF (VEGF-A) induces hem- and lymphangiogenesis by binding to VEGF receptor (VEGFR)-1 and -2. VEGF-B reacts only with VEGFR1. The lymphangiogenic molecules VEGF-C and VEGF-D both bind to VEGFR2 and VEGFR3 (for review see Ref. 12). In tumor hemangiogenesis as well as in other condi- 
tions of hypoxic and inflammatory hemangiogenesis, VEGF-A through VEGFR2-ligation has emerged as the main growth factor that induces hemangiogenesis. ${ }^{12}$

Using the mouse model of normal-risk keratoplasty, the present study analyzed (1) whether lymphangiogenesis accompanies hemangiogenesis after normal-risk keratoplasty, (2) the time course of blood and lymphatic vessel outgrowth after keratoplasty, (3) whether there is a difference in postkeratoplasty angiogenesis between syngeneic and allogeneic grafting, and (4) whether inhibition of hem- and lymphangiogenesis by a molecular trap designed to eliminate VEGF-A (VEGF $\operatorname{Trap}_{\mathrm{R} 1 \mathrm{R} 2}$ ) promotes long-term graft survival in the normal-risk keratoplasty setting.

\section{MeTHODS}

\section{Mice and Anesthesia}

Six- to 8-week-old male C57BL/6 mice were used as donors, and same-aged male BALB/c mice (Taconic, Germantown, NY) as recipients in the mouse model of normal-risk keratoplasty. ${ }^{13}$ For syngeneic transplantations, 6- to 8-week-old male BALB/c mice were used both as donors and as recipients. For the dose-response studies, 8-week-old male C57BL/6 mice were used. All animals were treated in accordance with the ARVO Statement for the Use of Animals in Ophthalmic and Vision Research. Mice were anesthetized using a mixture of ketamine and xylazine $(120 \mathrm{mg} / \mathrm{kg}$ and $20 \mathrm{mg} / \mathrm{kg}$ body weight, respectively).

\section{Dose-Response of VEGF Trap $\operatorname{RrR2}_{2}$}

To establish the minimum dose of VEGF Trap ${ }_{\mathrm{R} 1 \mathrm{R} 2}$, a molecular trap for VEGF-A (described later), that would effectively suppress corneal neovascularization for at least 1 week, five different doses of VEGF $\operatorname{Trap}_{\mathrm{R} 1 \mathrm{R} 2}$ were tested in mice, which received three interrupted intrastromal sutures (10-0 nylon, $50 \mu \mathrm{m}$-diameter; Sharpoint, Surgical Specialties Corp., Reading, PA; $n=5$ mice per dosage). Gentamicin and ophthalmic ointment were applied immediately after surgery. After surgery (day 0), mice received a single subcutaneous injection of VEGF $\operatorname{Trap}_{\mathrm{R} 1 \mathrm{R} 2}(25,12.5,6.25,2.5$ or $0.5 \mathrm{mg} / \mathrm{kg})$ or human Fc $(12.5 \mathrm{mg} / \mathrm{kg}$; control). Corneas were harvested on day 9 after suture placement, after an intravenous administration of an endothelium-specific fluoresceinconjugated lectin (Lycopersicon esculentum; Vector Laboratories, Burlingame, CA). The isolated corneas were flatmounted on glass slides, and images of lectin-labeled vessels were captured with a digital camera (Spot RT; Diagnostic Instruments, Inc., Sterling Heights, MI) attached to a microscope (Microphot-FXA; Nikon Inc., Garden City, NY). Image-analysis software (Image 1.62c; Scion Corporation, Frederick, $\mathrm{MD})$ was used to quantify the extent of corneal neovascularization.

\section{Corneal Transplantation in Mice}

Orthotopic corneal allografting in the mouse model of normal-risk keratoplasty was performed as described previously. ${ }^{13}$ Donor corneas were excised by trephination using a $2.0 \mathrm{~mm}$ bore and cut with curved Vannas scissors. Until grafting, corneal tissue was placed in chilled phosphate-buffered saline (PBS). Recipients were anesthetized, and the graft bed was prepared by trephining a $1.5-\mathrm{mm}$ site in the central cornea of the right eye and discarding the excised cornea. The donor cornea was immediately applied to the bed and secured in place with eight interrupted sutures (11-0 nylon, 70- $\mu \mathrm{m}$ diameter needles; Arosurgical, Newport Beach, CA). Antibiotic ointment (Oxymycin; Pharmafair, Hauppauge, NY) was placed on the corneal surface and the eyelids sutured with 8-0 suture (Sharpoint; Surgical Specialties Corp.). Recipients of grafts in which bleeding developed in the immediate postoperative period were discarded from further evaluation. All grafted eyes were examined after 72 hours, and grafts with technical difficulties (hyphema, cataract, infection, loss of anterior chamber) were excluded from further consideration. Tarsorrhaphy and corneal sutures were removed after 7 days, and grafts were then examined at least twice a week until week 8 after transplantation by slit lamp microscopy and scored for opacity as described previously. ${ }^{13}$ The survival experiment was performed twice and comprised 10 and 12 mice per experiment in both groups. Clinical scores of corneal grafts for opacity were as follows: 0 , clear; +1 , minimal, superficial (nonstromal) opacity; pupil margin and iris vessels readily visible through the cornea; +2 , minimal, deep (stromal) opacity; pupil margins and iris vessels visible; +3 , moderate stromal opacity; only pupil margin visible; +4 , intense stromal opacity; only a portion of pupil margin visible; and +5 , maximum stromal opacity; anterior chamber not visible. Grafts with opacity scores of +2 or greater after 2 weeks were considered to have been rejected. ${ }^{13}$ Syngeneic transplantations were performed and evaluated in a similar manner.

\section{Immunohistochemistry and Morphometry of Angiogenesis and Lymphangiogenesis in the Cornea}

Briefly, corneal flatmounts were rinsed in PBS, fixed in acetone, rinsed in PBS, blocked in $2 \%$ bovine serum albumin, stained with FITC-conjugated CD31/platelet-endothelial cell adhesion molecule (PECAM)-1 overnight (1:100; Santa Cruz Biotechnology, Santa Cruz, CA), washed, blocked, stained with LYVE-1 (1:500; a lymphatic endothelium-specific hyaluronic acid receptor), ${ }^{6,14}$ washed, blocked, and stained with Cy3 (1:100; Jackson ImmunoResearch Laboratories, West Grove, PA), and analyzed by microscope (Axiophot; Carl Zeiss Meditec). Digital pictures of the flatmounts were taken with an imageanalysis system (Spot; Diagnostic Instruments). Then, the area covered by $\mathrm{CD} 31^{3+} / \mathrm{LYVE}^{-} 1^{-}$blood vessels and $\mathrm{CD} 31^{+} / \mathrm{LYVE}-1^{3+}$ lymph vessels $^{6}$ was measured morphometrically on the flatmounts with NIH Image software (available by ftp at zippy.nimh.nih.gov/ or at http:// rsb.info.nih.gov/nih-image; developed by Wayne Rasband, National Institutes of Health, Bethesda, MD). The total corneal area was outlined, with the innermost vessel of the limbal arcade serving as the border. The total area of blood versus lymphatic neovascularization was then normalized to the total corneal area and the percentage of the cornea covered by each vessel type calculated.

\section{Neutralization of VEGF-A with a Cytokine Trap: VEGF Trap $_{\text {R1R2 }}$}

A newly designed molecular trap for VEGF-A, VEGF Trap ${ }_{\mathrm{R} 1 \mathrm{R} 2}$, comprising the receptor binding domains of VEGF receptor 1 and 2 coupled to a human Fc fragment (Regeneron Pharmaceuticals Inc., Tarrytown, $\mathrm{NY})^{15}$ was used in the transplant survival experiment at a concentration of $12.5 \mathrm{mg} / \mathrm{kg}$ intraperitoneally (IP) at time of surgery (CHO hVEGFR1 [Ig domain 2], R2 [Ig domain 3]-Fc), and 3, 7, and 14 days after surgery. ${ }^{15}$ Human Fc-fragment given IP at same concentration and times was used in the control mice ( $\mathrm{sCHO} \mathrm{h} \mathrm{Fc}$ ).

\section{Statistical Analysis}

Statistical significance was analyzed by the Mann-Whitney test. Differences were considered significant at $P<0.05$. Each experiment was performed at least twice with similar results. Graphs were drawn by computer (Prism, ver. 3.02; Graph Pad, San Diego, CA).

\section{Results}

\section{Dose-Response of Angiogenesis Inhibition by VEGF Trap $_{\text {R1R2 }}$}

As shown in Figure 1, VEGF Trap $\mathrm{R} 1 \mathrm{R} 2$, at doses of 25 or 12.5 $\mathrm{mg} / \mathrm{kg}$, completely inhibited suture-induced inflammatory corneal neovascularization. In contrast, doses of 6.25 and 2.5 $\mathrm{mg} / \mathrm{kg}$ produced $\sim 50 \%$ and $\sim 20 \%$ inhibition of corneal neovascularization, respectively, whereas the lowest dose tested, $0.5 \mathrm{mg} / \mathrm{kg}$, had a negligible effect ( $<5 \%$ inhibition). Therefore, 

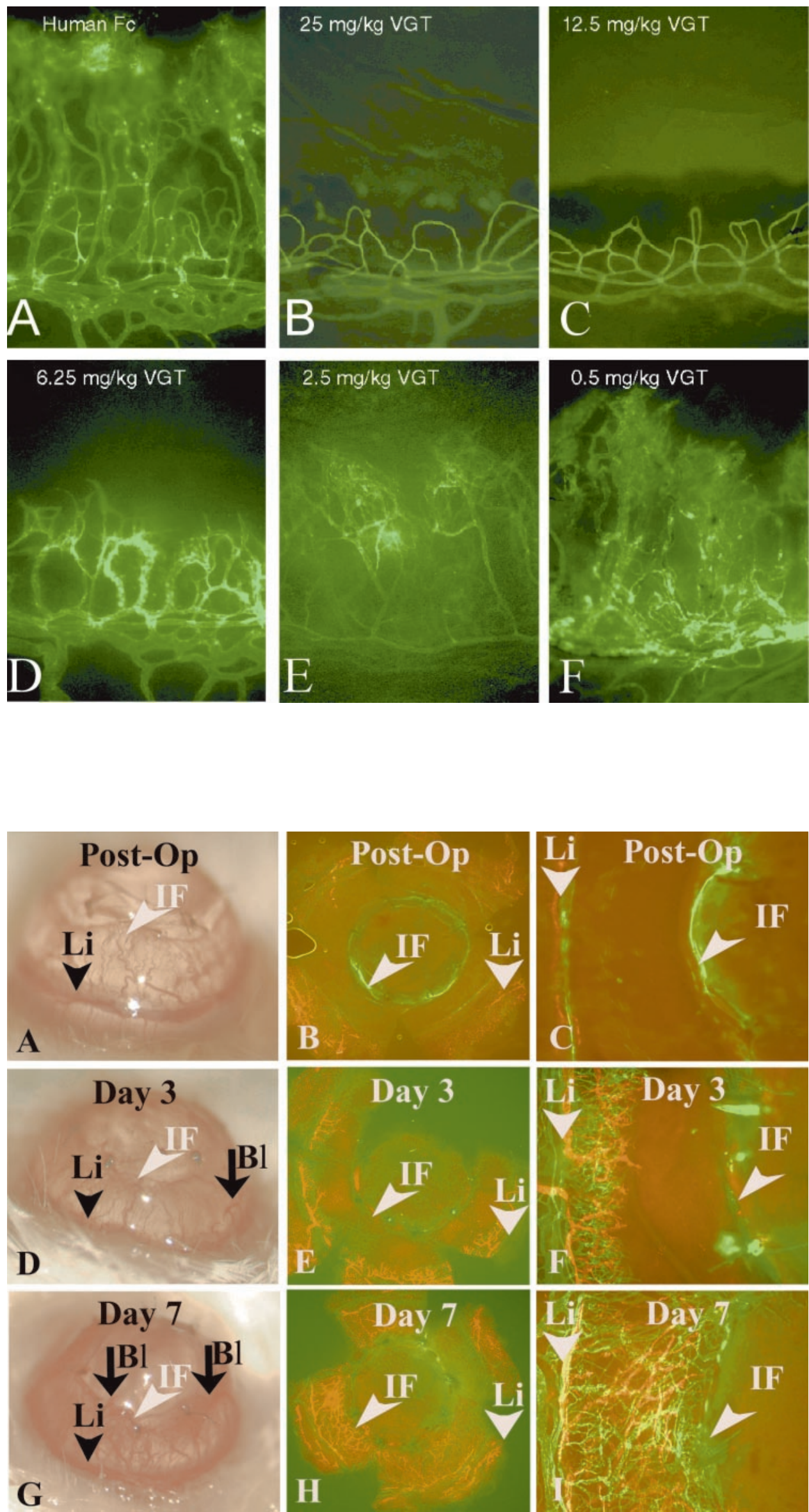

FigURE 1. Dose-response of the antiangiogenic effect of VEGF Trap $\mathrm{R} 1 \mathrm{R} 2$. Immediately after placement of intrastromal corneal sutures, mice received human Fc protein (control: A) or 25 (B), $12.5(\mathbf{C}), 6.25$ (D), 2.5 (E),

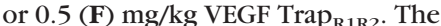
dose of $12.5 \mathrm{mg} / \mathrm{kg}$ was the lowest that provided complete inhibition of suture-induced corneal neovascularization (as measured in lectin-stained corneal flatmounts 9 days after suture placement; the limbal vascular arcade is located at the bottom of each image). Magnification, $\times 100$.

Figure 2. Early, combined induction of hem- and lymphangiogenesis after normal-risk allogeneic keratoplasty. There was neither biomicroscopically (A) nor immunohistochemically (B/C: $\mathrm{CD}^{+} 1^{+}$blood vessels: green; LYVE-1 ${ }^{+}$lymphatic vessels: red) detectable hem- or lymphangiogenesis immediately after normal-risk allogeneic keratoplasty (B: corneal flatmount; C: detail from B). By day 3 after surgery (D-F), corneal blood vessels (Bl) grew into the avascular recipient beds. Immunostaining revealed new blood vessels to be accompanied by lymphatic vessels (E, F: red vessels). Both vessel types penetrated approximately $30 \%$ to $50 \%$ from the limbus to the graft bed. One week after normal-risk keratoplasty (G-I) both vessels types had already reached donor tissue and spread along the interface $(\mathbf{H}, \mathbf{I})$, but these vessels rarely invade donor tissue. Li, limbal vascular arcade; IF, interface. 

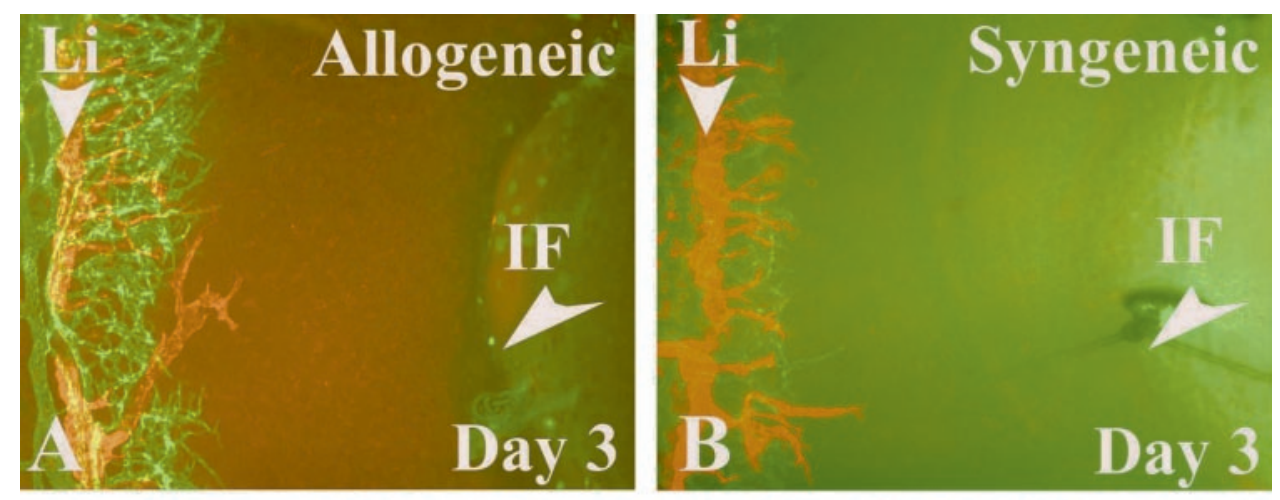

FIGURE 3. Combined induction of
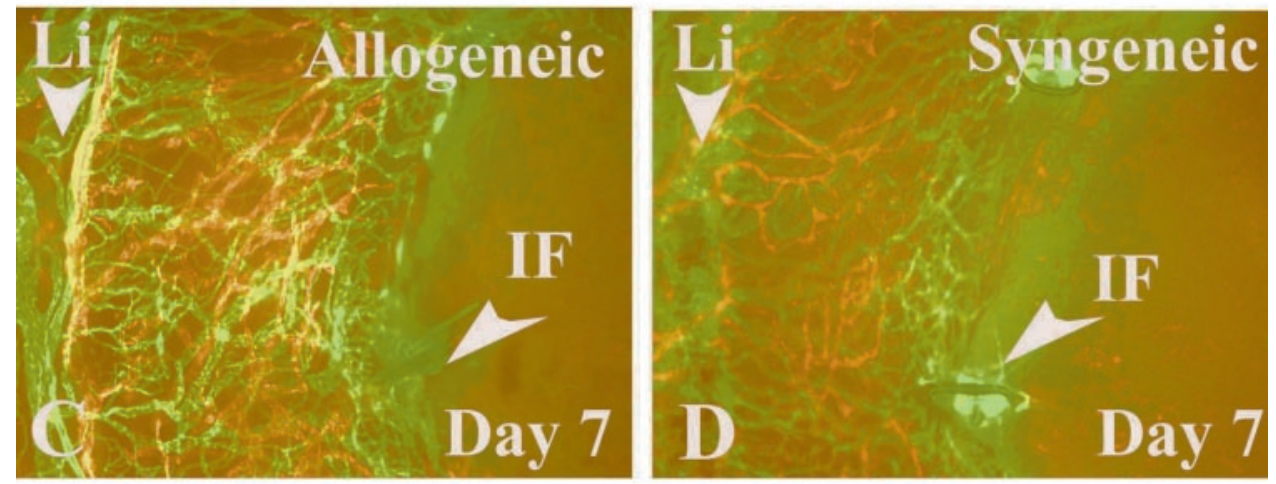

hem- and lymphangiogenesis after allogeneic and syngeneic keratoplasty. Allogeneic cornea grafts (A, C: $\mathrm{C} 57 \mathrm{BL} / 6$ to $\mathrm{BALB} / \mathrm{c}$ ) and syngeneic corneal grafts (B, D: BALB/c to $\mathrm{BALB} / \mathrm{c}$ ) were compared. The micrographs depict representative segments from corneal flatmounts at days $3(\mathbf{A}, \mathbf{B})$ and 7 (C, D) after grafting. The limbal vascular arcade (Li) is at the left; the graft-bed-interface (IF) is at the right. (E) Morphometric comparison reveals no significant differences between allo- and syngeneic grafting with respect to hem- and lymphangiogenesis (either at day 3 [shown] or at day 7 [not shown]; $n=$ 8 mice per group).

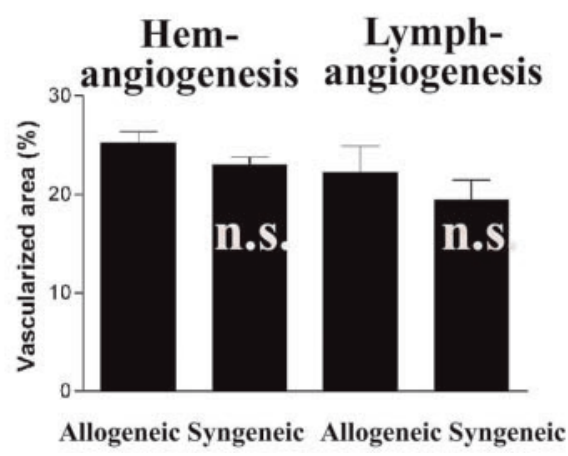

for subsequent experiments, a dose of $12.5 \mathrm{mg} / \mathrm{kg}$ VEGF Trap $_{\mathrm{R} 1 \mathrm{R} 2}$ was chosen.

\section{Rapid and Parallel Onset of Hemangiogenesis and Lymphangiogenesis after Normal-Risk Allogeneic Corneal Transplantation}

To determine whether the mild and temporary hemangiogenesis occurring after normal-risk keratoplasty is accompanied by lymphatic vessel outgrowth from the limbus into the normally alymphatic cornea, we studied the time course of ingrowth of both vessel types at days $0,3,7,14,21$, and 28 after allogeneic keratoplasty (only accepted grafts). Immediately after surgery, blood, and lymphatic vessels were not detectable either in the host or in donor tissue using biomicroscopy and immunohistochemistry on corneal flatmounts (Fig. 2). But, at day 3 after allografting, both methods revealed new blood vessels growing into the cornea already one third to one half the way toward the graft interface. By day 7 , these vessels had usually reached the donor tissue, but they rarely invaded the donor tissue itself. Analyzing flatmounts stained with LYVE-1 as a lymphatic vessel-specific marker showed that $\mathrm{CD} 31^{3+} / \mathrm{LYVE}-1^{-}$blood vessels were regularly accompanied by LYVE- $1^{3+} / \mathrm{CD} 31^{+}$lym-

phatic vessels (Fig. 2). Both vessel types reached the interface simultaneously at day 7 . Thereafter, coincident with suture removal, both vessel types started to regress (if no immune rejection occurred; data not shown).

\section{Difference in Postkeratoplasty Hem- and Lymphangiogenesis between Syngeneic and Allogeneic Corneal Transplantation}

To determine whether the simultaneous induction of hem- and lymphangiogenesis after normal-risk keratoplasty is primarily an effect of the surgical trauma, suturing, and wound-healing processes or is secondary to early immunologic rejection reactions, we compared the speed and extent of both hem- and lymphangiogenesis occurring after keratoplasty between allogeneic $(\mathrm{C} 57 \mathrm{BL} / 6$ into $\mathrm{BALB} / \mathrm{c})$ and syngeneic grafts $(\mathrm{BALB} / \mathrm{c}$ into BALB/C) at days 3, 7, 14, 21, and 28 after transplantation (Fig. 3). In both groups, blood and lymphatic vessels grew out after keratoplasty and by day 3 reached approximately one third to one half of the limbus-interface distance. At day 7 after syngeneic and allogeneic grafting, both vessel types had reached the interface, before they started to regress. Furthermore, there was no significant difference in the hem- and 

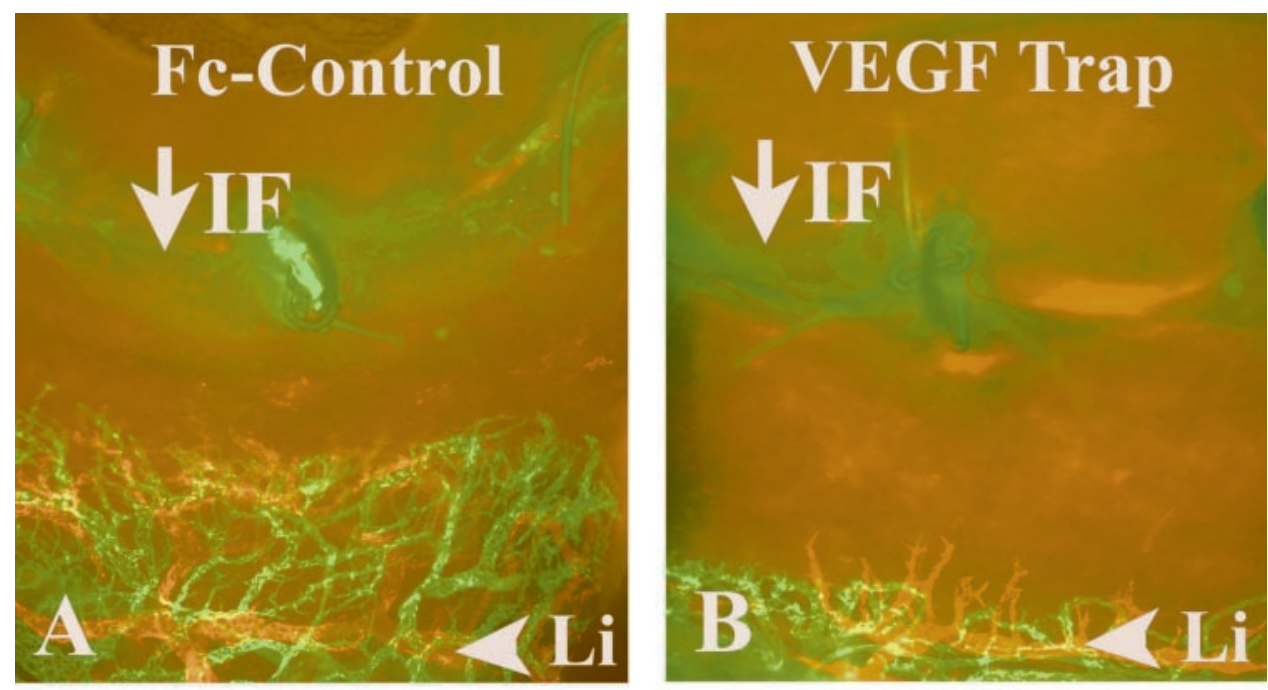

\section{Hem- Lymph- angiogenesis angiogenesis}

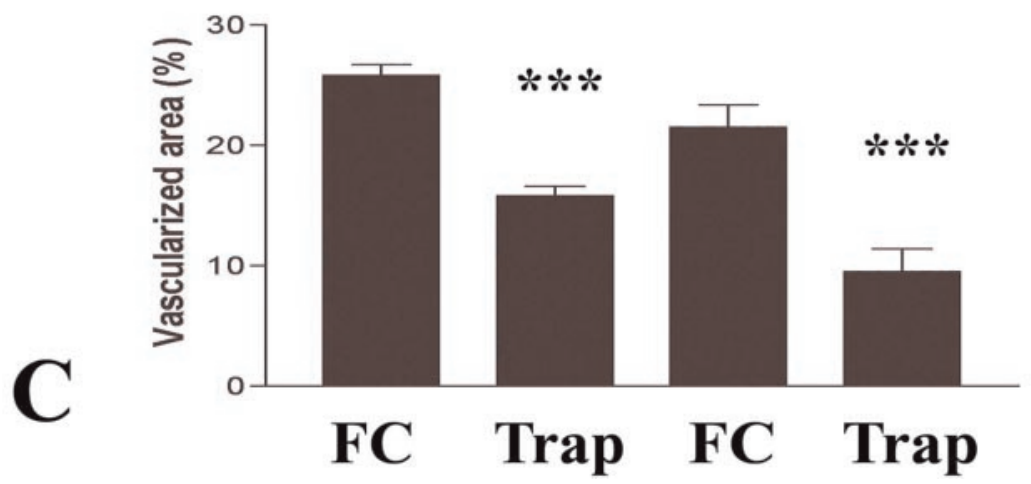

FIGURE 4. Effect of pharmacologic neutralization of VEGF-A on hem- and lymphangiogenesis after normal-risk allogeneic keratoplasty. Compared with the Fc-treated control (A), VEGF-A neutralization using VEGF $\operatorname{Trap}_{\mathrm{R} 1 \mathrm{R} 2}(\mathbf{B})$ inhibited both clinically visible hemangiogenesis (green) as well as biomicroscopically invisible LYVE- $^{+}$lymphangiogenesis (red; shown as detail from corneal flatmounts; between donor at bottom and host at top). (C) Morphometry at day 3 after penetrating keratoplasty demonstrates significant inhibition of both hem- and lymphangiogenesis by VEGF-A neutralization $(P<0.001 ; n$ $=6$ per group). Li, limbus; IF, interface. lymphovascularized area, comparing syngeneic and allogeneic grafts at 3 days (allogeneic with hemovascularized area [HA] $25.2 \% \pm 4.1 \%$ and lymphovascularized area [LA] $22.2 \% \pm 9.4 \%$ vs. syngeneic HA: $23 \% \pm 2.7 \%$ and LA $19.4 \% \pm 7.2 \%)$ and 7 days (allogeneic HA: $53.8 \% \pm 11.2 \%$ and LA: $37.9 \% \pm 6.2 \%$ vs. syngeneic HA: $55.9 \% \pm 8.2 \%$ and LA: $38 \% \pm 22.7 \%$ ) after surgery ( $n=8$ mice per group per time point).

\section{Effect of Neutralization of VEGF-A after Normal- Risk Keratoplasty on Postoperative Hemangiogenesis and Lymphangiogenesis}

To determine the extent to which combined hem- and lymphangiogenesis occurring after keratoplasty depends on VEGF-A, we analyzed the effect of pharmacological neutralization of VEGF-A using a novel cytokine trap (VEGF $\left.\operatorname{Trap}_{\mathrm{R} 1 \mathrm{R} 2}\right) .{ }^{15,16}$ Mice received either intraperitoneal injections of VEGF Trap R1R2 $_{2}(12.5 \mathrm{mg} / \mathrm{kg})$ at surgery and 3 days later. Control animals received the Fc-protein in the same dosage. At day 3 and 7 after surgery, the extent of hem- and lymphangiogenesis was compared between these two groups ( $n=6$ mice per group per time point). At days 3 and 7 after surgery, the hemovascularized area was significantly smaller in trap-treated mice (day 3: $15.8 \% \pm 4.0 \%$; day $7: 25.2 \% \pm 13.3 \%$ ) compared with mice receiving only the Fc-fragment (day 3: $25.8 \% \pm$ $4.4 \%$; day $7: 48.3 \% \pm 12.8 \% ; P<0.0001 ;$ Fig. 4$)$. This was also true of the lymphovascularized area comparing Trap- $(9.5 \pm$
9.4\%) and Fc-treated mice on day $3(21.5 \% \pm 9.3 \% ; P<$ $0.0001)$. At day 7 , the lymphovascularized area was smaller, but not significantly different in the Trap-group $(28.7 \% \pm$ $20.3 \%)$ compared with the Fc-group $(51.5 \% \pm 23.8 \% ; P=$ $0.06)$. In contrast to results obtained in corneal injury models (Cao et al., manuscript submitted) ${ }^{16}$ neither hem- or lymphangiogenesis was completely inhibited by the VEGF Trap R1R2 $_{2}$ after corneal transplantation. However, the number of lymphatic vessels reaching the graft-host interface $(10.6 \pm 0.6$ vs. $1.3 \pm 1.5$ vessels) and the number of hours that the interface was filled with draining lymphatic vessels were much more in the Fc-treated than in the Trap-treated group at day 7 ( $3 \pm 2$ vs. $0.2 \pm 0.3$ hours; not significant due to small sample size). This may indicate that lymphovascularized area per se is less decisive for host sensitization than the contact area with donor tissue (described later).

\section{Effect on Graft Survival of Partial Inhibition of Early Postoperative Hem- and Lymphangiogenesis by Trapping VEGF-A after Normal-Risk Surgery}

Because hem- and lymphangiogenesis that occurred after normal-risk keratoplasty peaked around day 7 , and regressed thereafter, and because both vascular processes could be significantly inhibited by early postoperative neutralization of 


\section{Survival proportions}

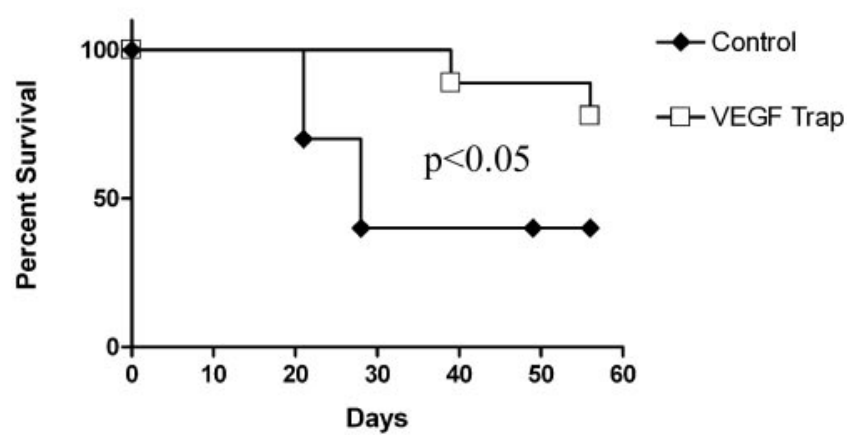

FIGURE 5. Effect of pharmacologic neutralization of VEGF-A on survival of allogeneic cornea grafts. Panels of $\mathrm{BALB} / \mathrm{c}$ mice received orthotopic transplants from $\mathrm{C} 57 \mathrm{BL} / 6$ donors in one low-risk eye. The recipients in one panel were treated with VEGF Trap ${ }_{\mathrm{R} 1 \mathrm{R} 2}$, whereas the other panel (control) received Fc-fragments only. Survival of grafts in mice treated with VEGF Trap was significantly greater than in control animals (78\% vs. $40 \% ; P<0.05 ; n=22$ mice in both groups).

VEGF-A, we determined whether inhibition of postkeratoplasty hem- and lymphangiogenesis during this interval improved graft survival. The long-term survival of $\mathrm{C} 57 \mathrm{BL} / 6$ grafts placed into avascular BALB/c recipient beds was compared between mice receiving an IP injection of $12.5 \mathrm{mg} / \mathrm{kg}$ VEGF Trap $\mathrm{R} 1 \mathrm{R} 2_{2}$ and those receiving Fc-fragment alone, at surgery and 3, 7, and 14 days later. As Figure 5 shows, trapping of VEGF-A caused significantly improved long-term graft survival at 8 weeks after surgery $(78 \%)$, compared with grafts in eyes of Fc-treated control mice $(40 \% ; P=0.044 ; n=22$ in both groups).

\section{Discussion}

Whereas preexisting corneal blood vessels have long been established as risk factors for immune rejection after corneal transplantation, ${ }^{4,13}$ the pathogenesis, potential association with lymphangiogenesis, and immunologic importance of mild hemangiogenesis after normal-risk keratoplasty have yet to be determined. ${ }^{9-11}$ Using the mouse model of normal-risk keratoplasty, we provide novel evidence (1) that normal-risk keratoplasty itself promotes parallel and rapid outgrowth of both blood and lymphatic vessels into the avascular recipient bed; (2) that because there was no significant difference between postoperative hem- and lymphangiogenesis comparing syngeneic and allogeneic corneal grafting, early postoperative release of hem- and lymphangiogenic growth factors seems to be triggered mainly by surgical trauma, wound-healing, and corneal suturing rather than immune rejection; (3) that neutralization of VEGF-A after surgery not only inhibited hem- and lymphangiogenesis, but promoted long-term corneal allograft survival. The results establish hem- and lymphangiogenesis occurring after normal-risk keratoplasty as novel risk factors for subsequent immune rejections.

The molecular trap (VEGF $\operatorname{Trap}_{\mathrm{R} 1 \mathrm{R} 2}$ ) used in this study neutralized VEGF-A and PIGF with high affinity. Neutralization of VEGF-A has recently been shown to inhibit not only hemand lymphangiogenesis, but also to interfere with recruitment of inflammatory cells into the cornea (Cao J, et al., manuscript submitted). ${ }^{16}$ This effect of VEGF neutralization has been attributed to inhibition of neutrophil and macrophage chemotaxis mediated by ligation of VEGFR1 ${ }^{17,18}$ Trapping of VEGF-A thereby exerts direct and indirect antiangiogenic effects. Therefore, the graft survival-promoting effect of VEGF-A neutralization can also be attributed to multiple mechanisms. First, inhibition of hem- and lymphangiogenesis after keratoplasty interferes with the development of both an afferent (lymphatic vessels) and an efferent pathway (blood vessels) for a subsequent immune response. ${ }^{1,7}$ In addition, trapping of VEGF-A may impede the recruitment of APCs to the graft bed.

The relative importance of heme versus lymphangiogenesis after normal-risk keratoplasty for subsequent immune rejections remains unknown, because in this study both processes

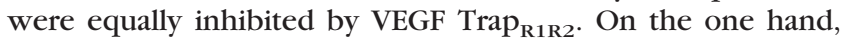
blood vessels reaching the graft are essential for delivery of APCs and alloreactive T-lymphocytes to the graft. On the other hand, lymphatic vessels seem to facilitate escape of APCs to regional lymph nodes, enhancing allosensitization. However, studies demonstrating that removal of regional lymph nodes can promote complete survival of corneal allografts placed in high- and normal-risk settings, ${ }^{19,20}$ and a study demonstrating increased transport of donor APCs to regional lymph nodes in inflamed (and probably lymphovascularized) beds, ${ }^{8}$ suggest that afferent corneal lymphatics that promote sensitization may be equal, or even more important than efferent corneal blood vessels that provide an entry route for immune effector cells.

Corneal allograft survival in the normal-risk mouse model $(\mathrm{C} 57 \mathrm{BL} / 6$ to $\mathrm{BALB} / \mathrm{c})$ is reduced from around $50 \%$ after 8 weeks to $0 \%$ after 2 weeks, if the recipient bed is prevascularized. ${ }^{5,21}$ We have demonstrated parallel outgrowth of both blood and lymphatic vessels in this model, ${ }^{16}$ implying that donor tissue has immediate access to draining host lymphatic vessels after high-risk grafting and is exposed to efferent host blood vessels. Because we demonstrated in the current study that 1 week after normal-risk keratoplasty both vessels types also reached donor tissue, the question arises of why the survival rates between $\mathrm{C} 57 \mathrm{BL} / 6$ grafts placed into avascular, but neovascularizing versus already neovascularized graft beds, are so different. One explanation concerns the possibility of a time-dependent window of opportunity during which recipient sensitization to donor alloantigens after keratoplasty leads to graft rejection. Whereas grafts placed in high-risk eyes induce donor-specific sensitization promptly (within 7 days), ${ }^{5}$ presumably because antigens have access to draining lymph nodes through preestablished lymphatics, by contrast, allografts placed in low-risk eyes do not generate sensitization until 2 to 4 weeks after grafting, ${ }^{22}$ probably reflecting the time needed for lymphangiogenesis to develop. Once the drainage system is established, graft-derived antigens reach the local lymph node, and activate donor-specific alloreactive T-cells, which can cause rejection. If, however, sensitized $\mathrm{T}$ cells disseminate only after 14 to 21 days, these effectors must compete with the regulatory T-cells of ACAID which begin to emerge at that time. ${ }^{23}$ Neutralization of VEGF-A at the time of surgery retards lymphangiogenesis in the graft bed, thus narrowing the window of opportunity during which recipient sensitization takes place and therefore may reflect a shift in the balance of the recipient alloimmune response toward acceptance (ACAID) rather than rejection. This idea is compatible with the observation that a temporary depletion of local macrophages by subconjunctival injection of clodronate liposomes at the time of keratoplasty in low-risk eyes achieves permanent survival of most of these grafts. ${ }^{24-25}$ Other possible explanations include a role for the degree of antigen flow, the APC phenotype, and other related or unrelated differences between these graft types.

Inhibition of both hem- and lymphangiogenesis by neutralization of VEGF-A was incomplete in this study of keratoplasty, whereas the same dosage of VEGF Trap in a previous study completely inhibited both angiogenic processes after corneal suturing. ${ }^{16}$ This may suggest that the release of angiogenic factors after corneal transplantation is greater than after suture 
placement alone, and that the present dosing regimen is insufficient for complete suppression of angiogenesis in this context. Alternatively, because lymphangiogenesis is thought to be mediated mainly by VEGF-C and -D binding to their high-affinity receptor VEGFR3 on lymphatic vascular endothelium, ${ }^{12,26-29}$ and because the VEGF Trap $_{\mathrm{R} 1 \mathrm{R} 2}$ used in this study does not bind VEGF-C and -D, ${ }^{16}$ adding VEGFR3-signaling inhibitors to the treatment regimen may more completely inhibit lymphangiogenesis and further improve graft survival after normalrisk keratoplasty. The fact that pharmacological neutralization of VEGF-A, which is mainly thought of as a hemangiogenic growth factor, ${ }^{12,26-29}$ significantly inhibited lymphangiogenesis, suggests a novel, important role for VEGF-A in generating lymphangiogenesis and in promoting sensitization to donor antigens. In line with this interpretation, an important role for VEGF-A in another transplant setting was recently demonstrated. ${ }^{30}$ For human cardiac allografts a correlation between increased intragraft VEGF-levels, inflammatory cell influx and all grades of acute rejection was shown. ${ }^{30}$ It has been reported that topically applied anti-VEGF antibodies reduced the degree of inflammation and hemangiogenesis in the rat model of high-risk keratoplasty (Lewis to Fisher rats), ${ }^{31}$ and could improve short-term survival of grafts in this high-risk model. ${ }^{31}$ The occurrence of lymphangiogenesis or the effect of inhibiting hem- and lymphangiogenesis on long-term survival were not analyzed in this study. ${ }^{31}$

Our finding that there was no difference in early postoperative hem- and lymphangiogenesis after syngeneic versus allogeneic grafting suggests an important role of surgery and surgery-related wound healing in inducing these vascular responses, rather than immunologic mechanisms. This is in line with a previous study in humans in which the degree of postkeratoplasty hemangiogenesis was significantly lower in patients after nonmechanical excimer laser trephination (which induces less vigorous wound healing) than after mechanical trephination. ${ }^{9}$ Taken together, the evidence suggests a novel role of surgery/wound healing itself in determining the immunologic fate of corneal grafts and a close association of immune and angiogenic responses in the cornea. $^{32}$

Thinking about translating the results obtained in our study to the clinical setting, one has to keep in mind that important differences exist between penetrating keratoplasty in humans and in the mouse model: continuous suturing in human lowrisk patients versus interrupted sutures in mouse surgery, suture placement for over 1 year in patients compared with 1 week in mice and longer distances between interface and vessels at the limbus in patients compared with mice, for example. Therefore, because our results establish hem- and lymphangiogenesis postkeratoplasty as novel risk factors for subsequent immune rejections even after normal-risk transplantation in the mouse model, it seems reasonable to determine whether this association also holds true for patients, whether there is postkeratoplasty lymphangiogenesis in humans, and when the association is confirmed in patients, to try to inhibit postkeratoplasty neovascularization and improve graft survival.

\section{Acknowledgments}

The authors thank colleagues at the Schepens Eye Research Institute, especially Jackie Doherty for general support, Don Pottle for help with confocal and immunofluorescent imaging and Stephanie Caroll and Marie Ortega for help with animal housing in the vivarium.

\section{References}

1. Streilein JW, Yamada J, Dana MR, Ksander BR. Anterior chamberassociated immune deviation, ocular immune privilege, and orthotopic corneal allografts. Transplant Proc. 1999;31:1472-1475.

2. Küchle M, Cursiefen C, Nguyen NX, et al. Risk factors for corneal allograft rejection: intermediate results of a prospective normalrisk keratoplasty study. Graefes Arch Clin Exp Ophthalmol. 2002; 240:580-584.

3. Dana MR, Streilein JW. Loss and restoration of immune privilege in eyes with corneal neovascularization. Invest Ophthalmol Vis Sci. 1996;37:2485-2494.

4. Maguire MG, Stark WJ, Gottsch JD, et al. Risk factors for corneal graft failure and rejection in the collaborative corneal transplantation studies: collaborative Corneal Transplantation Studies Research Group. Ophthalmology. 1994;101:1536-1547.

5. Sano Y, Ksander BR, Streilein JW. Fate of orthotopic corneal allografts in eyes that cannot support anterior chamber-associated immune deviation induction. Invest Ophthalmol Vis Sci. 1995;36: 2176-2185

6. Cursiefen C, Schlötzer-Schrehardt U, Küchle M, et al. Lymphatic vessels in vascularized human corneas: immunohistochemical investigation using LYVE-1 and podoplanin. Invest Ophthalmol Vis Sci. 2002; 43:2127-2135.

7. Cursiefen C, Chen L, Dana MR, Streilein JW. Corneal lymphangiogenesis: evidence, mechanisms, and implications for corneal transplant immunology. Cornea. 2003;22:273-281.

8. Liu Y, Hamrah P, Zhang Q, Taylor AW, Dana MR. Draining lymph nodes of corneal transplant hosts exhibit evidence for donor major histocompatibility complex (MHC) class II-positive dendritic cells derived from MHC class II-negative grafts. J Exp Med. 2002;195: $259-268$.

9. Cursiefen C, Martus P, Nguyen NX, Langenbucher A, Seitz B, Küchle M. Corneal neovascularization after nonmechanical versus mechanical corneal trephination for non-high-risk keratoplasty. Cornea. 2002;21:648-652.

10. Dana MR, Schaumberg DA, Kowal VO, et al. Corneal neovascularization after penetrating keratoplasty. Cornea. 1995;14:604-609.

11. Cursiefen $C$, Wenkel $H$, Martus $P$, et al. Impact of short-term versus long-term topical steroids on corneal neovascularization after nonhigh-risk keratoplasty. Graefes Arch Clin Exp Ophthalmol. 2001; 239:514-521.

12. Carmeliet $\mathrm{P}$, Jain RK. Angiogenesis in cancer and other diseases. Nature. 2000;407:249-257.

13. Sonoda Y, Streilein JW. Orthotopic corneal transplantation in mice-evidence that the immunogenetic rules of rejection do not apply. Transplantation. 1992;54:694-704.

14. Banerji S, Ni J, Wang SX, et al. LYVE-1, a new homologue of the CD44 glycoprotein, is a lymph-specific receptor for hyaluronan. J Cell Biol. 1999;144:789-801.

15. Holash J, Davis S, Papadopoulos N, et al. VEGF-Trap: a VEGF blocker with potent antitumor effects. Proc Natl Acad Sci USA. 2002;99:11393-11398.

16. Cursiefen C, Chen L, Borges L, et al. VEGF-A stimulates lymph- and hemangiogenesis in inflammatory neovascularization via macrophage recruitment. J Clin Invest. 2004;113:1040-1050.

17. Shen H, Clauss M, Ryan J, et al. Characterization of vascular permeability factor/vascular endothelial growth factor receptors on mononuclear phagocytes. Blood. 1993;81:2767-2773.

18. Lee TH, Avraham H, Lee SH, Avraham S. Vascular endothelial growth factor modulates neutrophil transendothelial migration via up-regulation of interleukin-8 in human brain microvascular endothelial cells. J Biol Chem. 2002;277:10445-10451.

19. Yamagami S, Dana MR. The critical role of lymph nodes in corneal alloimmunization and graft rejection. Invest Ophthalmol Vis Sci. 2001;42:1293-1298.

20. Yamagami S, Dana MR, Tsuru T. Draining lymph nodes play an essential role in alloimmunity generated in response to high-risk corneal transplantation. Cornea. 2002;21:405-409.

21. Streilein JW, Bradley D, Sano Y, Sonoda Y. Immunosuppressive properties of tissues obtained from eyes with experimentally manipulated corneas. Invest Ophthalmol Vis Sci. 1996;37:413-424. 
22. Sonoda Y, Sano Y, Ksander B, Streilein JW. Characterization of cell-mediated immune responses elicited by orthotopic corneal allografts in mice. Invest Ophthalmol Vis Sci. 1995;36:427-434.

23. Sonoda Y, Streilein JW. Impaired cell-mediated immunity in mice bearing healthy orthotopic corneal allografts. J Immunol. 1993; 150:1727-1734.

24. Slegers TP, van der Gaag R, van Rooijen N, van Rij G, Streilein JW. Effect of local macrophage depletion on cellular immunity and tolerance evoked by corneal allografts. Curr Eye Res. 2003;26:7379.

25. Slegers TP, van Rooijen N, van Rij G, van der Gaag R. Delayed graft rejection in pre-vascularised corneas after subconjunctival injection of clodronate liposomes. Curr Eye Res. 2000;20:322-324.

26. Veikkola T, Jussila L, Makinen T, et al. Signalling via vascular endothelial growth factor receptor-3 is sufficient for lymphangiogenesis in transgenic mice. EMBO J. 2001;20:1223-1231.

27. Kubo H, Cao R, Brakenhielm E, Makinen T, Cao Y, Alitalo K. Blockade of vascular endothelial growth factor receptor-3 signaling inhibits fibroblast growth factor-2-induced lymphangio- genesis in mouse cornea. Proc Natl Acad Sci USA. 2002;99: $8868-8873$.

28. Karkkainen MJ, Petrova TV. Vascular endothelial growth factor receptors in the regulation of angiogenesis and lymphangiogenesis. Oncogene. 2000;19:5598-5605.

29. Nagy JA, Vasile E, Feng D, et al. Vascular permeability factor/ vascular endothelial growth factor induces lymphangiogenesis as well as angiogenesis. $J$ Exp Med. 2002;196:1497-1506.

30. Reinders MEJ, Fang JC, Wong W, Ganz P, Briscoe DM. Expression patterns of vascular endothelial growth factor in human cardiac allografts: association with rejection. Transplantation. 2003;76: 224-230.

31. Yatoh S, Kawakami Y, Imai M, et al. Effect of a topically applied neutralizing antibody against vascular endothelial growth factor on corneal allograft rejection of rat. Transplantation. 1998;66:15191524.

32. Cursiefen C, Masli S, Ng TF, et al. Roles of thrombospondin-1 and -2 in regulating corneal and iris angiogenesis. Invest Ophthalmol Vis Sci. 2004;45:1117-1124. 